\title{
EPR Paradox Solved by Special Theory of Relativity
}

\author{
J. LEE
}

\author{
17161 Alva Rd. \#1123, San Diego, CA 92127, U.S.A.
}

(Received November 7, 2013)

This paper uses the special theory of relativity to introduce a novel solution to Einstein-Podolsky-Rosen paradox. More specifically, the faster-than-light communication is described to explain two types of EPR paradox experiments: photon polarization and electron-positron pair spins. Most importantly, this paper explains why this faster-than-light communication does not violate the special theory of relativity.

DOI: 10.12693/APhysPolA.125.1107

PACS: 03.65.Ud

\section{Introduction}

EPR paradox refers to the thought experiment designed by Einstein, Podolsky, and Rosen (EPR) to show the incompleteness of wave function in quantum mechanics (QM) [1]. In QM, the Heisenberg uncertainty principle places a limitation on how precisely two complementary physical properties of a system can be measured simultaneously [2]. EPR came up with the following paradoxical scenario where the two properties, i.e. momentum and position, could be measured precisely, and thus would contradict the Heisenberg uncertainty principle.

In EPR paradox, two systems are initially allowed to interact (or entangled) with each other, and then they are separated apart to a far distance in order to prevent any further interaction. Next, a physical property, e.g. momentum of the first system is measured, which in turn can be used to determine the momentum of the second system. Now, if the position of the second system is measured subsequently, then the precise measurements of the two complementary properties would be achieved.

Then, in order for the uncertainty principle to be held true, it must mean that somehow the second system would have found out about the measuring event on the first system (so that the measurement on the second system would lose its precision in accordance to the uncertainty principle). But, if these two systems were sufficiently far apart from each other, then there could have been no way for the first system to have communicated any information to the second system in time. Therefore, EPR argued that this paradox had revealed the incompleteness of QM wave function [1].

In response to EPR paradox, Bell derived the inequality theorem regarding the probability for the local hidden variable theory, which was proposed by EPR as an explanation to the paradox [3]. Because this inequality value is different than the expected probability value of QM, an experiment can be designed to determine the validity of the hidden variable theory [3]. Many types of Bell's inequality experiments have been carried out thus far, and their results contradict the EPR hidden variable theory. The experimental results do, however, agree with Copenhagen interpretation of QM where an act of measurement on one of the two entangled systems causes the entangled wave function to collapse instantly, even when the two systems are at a great distance apart [4].
At this point, it is very important to note that both EPR paradox and Bell's inequality theorem assume that faster-than-light (FTL) communication between the two entangled systems is theoretically impossible $[1,3]$. It is the intent of this paper to show how the FTL communication could be possible without violating the special theory of relativity (SR). In short, it is the infinite time dilation that would be responsible for the instant FTL communication.

\section{Methods}

The two common types of Bell's inequality experiments are carried out by measurement of photon polarization and by measurement of electron-positron pair spins. Let us examine how the FTL communication could exist in these experiments.

\subsection{Photon polarization}

Bell's inequality experiment can be carried out by measuring the polarization of two entangled photons as shown below.

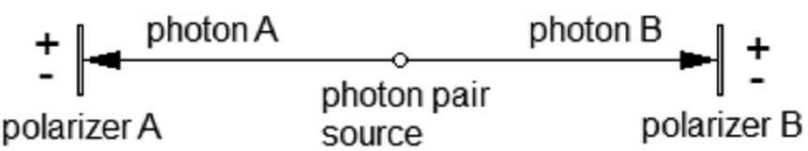

Fig. 1. EPR experiment with an entangled photon pair [5].

Figure 1 depicts a pair of entangled photons moving away from each other after being created together as a pair at the source location. Each photon can have either $(+)$ or $(-)$ state when measured by a polarizer. However, upon measurement, these two photons must possess the same states, e.g. if photon A is measured to have $(+)$ state, then photon $\mathrm{B}$ must have $(+)$ state, too; if $\mathrm{A}$ is $(-)$, then $\mathrm{B}$ is also $(-)$ [5].

The non-locality of quantum entanglement asserts that when photon A is measured, photon B would instantly find out about this measuring event on photon A, because their wave function collapses instantly upon the measurement [4].

But, what is the physical mechanism behind achieving this instant non-local action at a distance? To answer, we need to first understand how the time dilation works. In the SR, a moving clock runs slower than a stationary clock as shown below [6]:

$\Delta t^{\prime}=\Delta / \gamma$ 
where $\Delta t^{\prime}$ - time interval of a moving clock, $\Delta t$ - time interval of a stationary clock, $\gamma=1 /\left(1-v^{2} / c^{2}\right)^{1 / 2}-$ Lorentz factor, $v$ - velocity of moving clock, $c$ - speed of light.

For photon, which moves at the speed of light, it would experience an infinite time dilation - in other words, photon does not observe time passing at all; the time literally stands still in its inertial frame.

In our point of view, time passes by as photon is created in the past, and as it moves through in space in the present time. However, in photon's point of view, the time at its past creation is exactly the same time as the present. This means the photon at its past creation time is exactly the same, physically identical photon at the present time, and vice versa.

Now, let us get back to EPR paradox. When we measure the polarization of photon $\mathrm{A}$ at the present time, it determines the state of the present photon A. But, the photon $\mathrm{A}$ at the present time is exactly the same photon at its past creation time. Thus, whatever the determination made to the present photon $\mathrm{A}$ must have been made instantly to the past photon A at the creation time.

Next, because the past photon A at the creation time is now determined, consequently it determines the state of the past photon $\mathrm{B}$ at the exact same creation time. Using the same logic as above, the determination to the past photon $\mathrm{B}$ then equates to the instant determination to the present photon $\mathrm{B}$.

To summarize, measurement on the present photon $\mathrm{A}$ instantly determines the state of the both photon $\mathrm{A}$ and B.

Therefore the FTL communication can occur between the two entangled systems, and this invalidates the assumptions made by EPR paradox and Bell's inequality. Furthermore, this instant FTL communication explains the physical mechanism behind the Copenhagen interpretation of QM (instant collapse of wave function and non-locality).

Moreover, this FTL communication does not violate the SR. It does not violate the causality because the present determination cannot go any further past than the creation time of the photon, and the present determination can never tamper the past event. In order to tamper the past event, it would require a measurement in the past to detect the effects of the present determination; however, if such measurement were made in the past, then it would mean that the present measurement was not the first measurement, thus the present measurement would not have been able to make the determination to the photon to begin with. The very fact that the present measurement could make the determination implies that there was no past measurement. Because there was no past measurement, the past cannot be tampered by the present measurement.

Also, even though the FTL communication is made instantly at a great distance, it obeyed all rules set by the SR, i.e. no infinite energy was required because no physical entity traveled faster than the speed of light.

\subsection{Electron-positron spin}

Another way to test Bell's inequality is Bohm's version where spins of an electron-positron pair is measured. The underlying principle is very similar to the photon polarization experiment, except an electron-positron pair is used instead of a photon pair, and a spin is measured instead of the polarization [7].

Figure 2 depicts a pi meson decaying to create an electron-positron pair. The electron and positron move away in an opposite direction. The electron and positron can have either $(+)$ or $(-)$ spins. However, upon measurement, these two particles must possess the opposite spins, e.g. if the electron is measured to have $(+)$ spin, then the positron must have $(-)$ spin; if the electron has $(-)$, then the positron has $(+)[7]$.

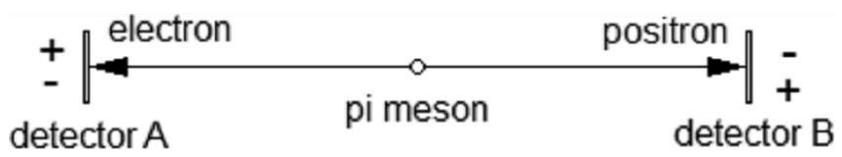

Fig. 2. EPR experiment with an entangled electronpositron pair [7].

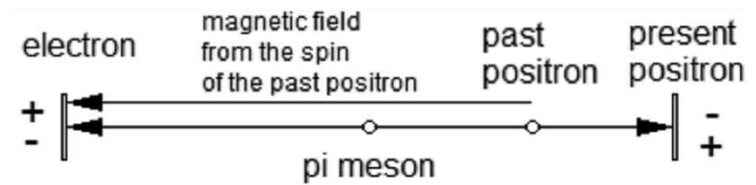

Fig. 3. The present electron is in contact with the magnetic field from the spin emitted from the positron at the past time.

Once more, in this experiment, the instant FTL communication would occur between the electron and positron upon a measurement of spin. The reasoning for the FTL communication is similar to the one given above for the photon experiment. The challenge is how to factor in the sub-light speed at which the electron and positron move, because their sub-light speed would indicate that their time dilation is not infinite like photon and thus they do experience time passing. Nevertheless, this challenge can be overcome when we realize that the electron and positron are always in contact with the magnetic fields produced by each other's spin, which does move at the speed of light. The spin of electron (as well as the spin of positron) produces the magnetic moment and emits the magnetic field [8].

Figure 3 depicts how the magnetic field from the spin emitted by the positron at some past time has traveled to the electron at the present time, when at that moment, the measurement is made against the electron spin. In fact, because the electron and positron are created at the same time at the same place, the electron would always be in contact with the magnetic field from the positron; and the positron in contact with the magnetic field from the electron.

Now, let us connect the dots for the FTL communication. The electron spin is measured at the present time. 
This determines the spin state of the present electron. Because the electron spin and the positron spin must correlate, the electron spin must correlate with the magnetic field of the positron spin as well. Thus, the state of the magnetic field of positron spin which is in contact with the electron, is also determined instantly. As previously mentioned, anything that moves with the speed of light does not experience time passing. Thus, this determination of the magnetic field state at the present electron position instantly causes the state of the magnetic field created by the past positron to be determined instantly. Consequently, the spin state of the past positron is determined instantly. Finally, if the past positron possessed a determined spin state, then the present positron must possess the same determined spin state, too.

To summarize, measurement on the spin state of the present electron instantly determines the spin state of the both electron and positron at the present time.

In the above experiment, we measured the spin state. But, as a matter of fact, it can be any two complementary physical properties. For example, how would measuring the precise position of electron would cause the FTL communication? We can use, e.g. gravitational field, to reason that the determination of the electron position would affect the determination of the position of the wave front of the gravitational field. Following the usual logic, then it would eventually lead to the instant determination of the positron position.

\section{Results}

The infinite time dilation of the SR causes the instant
FTL communication between the two entangled systems via light or any other field that travels at the speed of light. This FTL communication explains the physical mechanism behind the QM phenomena of the instant collapse of wave function and non-locality. Furthermore, this FTL communication does not violate the SR.

The assumption made by both EPR paradox and Bell's inequality, that it is impossible for two entangled systems to communicate with each other at a great distance, has been invalidated.

\section{Acknowledgments}

I would like to thank Jesus Christ for guidance and encouragement. I would like to thank Richard Lee for reviewing the paper.

\section{References}

[1] A. Einstein, B. Podolsky, N. Rosen, Phys. Rev. 47, 777 (1935).

[2] S. Gasiorowicz, Quantum Physics, 2nd ed., Wiley, New York 1996.

[3] J. Bell, Physics 1, 195 (1964).

[4] D. Dehlinger, M. Mitchell, Am. J. Phys. 70, 903 (2002).

[5] A. Aspect, Nature 398, 189 (1999).

[6] D.J. Griffiths, Introduction to Electrodynamics, 2nd ed., Prentice-Hall, Englewood Cliffs 1989.

[7] D.J. Griffiths, Introduction to Quantum Mechan$i c s$, 2nd ed., Pearson Education, Upper Saddle River 2005.

[8] R.A. Serway, Physics for Scientists and Engineers, 3rd ed., Saunders College Publ. Philadelphia 1990.

\section{Editorial comment on the paper by Justin Lee}

The Einstein-Podolsky-Rosen "paradox" does not constitute any paradox as long as we do not try do go beyond the probabilistic interpretation of quantum mechanics. In other words: there are no paradoxes as long as we assign a "wave function" or a "density matrix" to a statistical ensemble and not to a particular "sample" of this ensemble. This requirement is, however, a severe restriction on possible questions which can be legally asked within the theory. No wonder that there is a tendency to "revolt" against this restriction: one would like to think about wave function of a particular particle and not only about the quantum state of a source of radiation, producing these particles.

But, unfortunately, as soon as we leave this safe ground the paradoxes arise. Not only the EPR but also "Schrödinger cat" and others. Observe, however, that classical probability also displays such paradoxes as soon as we try to extend its validity beyond the probabilistic framework. For example, consider the 2-element probabilistic space whose points correspond to two possible results of drawing one card among the two: the red and the black. Suppose that after such a drawing you send by mail the withdrawn card to your friend in New York without checking the result of the drawing. The statistical state of such a system is obvious: with probability $\frac{1}{2}$ the red card is now in New York and the black one remains in Warsaw and with probability $\frac{1}{2}$ we deal with the inverse configuration.

What happens if, one year later, I check the colour of my card in Warsaw. If it is red, I immediately know that the black one is in New York and vice versa. Is there any paradox? Has any information been transmitted from New York to Warsaw with infinite speed? Has the probabilistic state $\left(\frac{1}{2}, \frac{1}{2}\right)$ been immediately reduced to the state $(0,1)$ ?

Quantum mechanics is much richer than that. In particular, there are experiments constructed as a chain of subsequent experiments (the particle first interacts with the wall and chooses which hole does it go, and then interacts with something else). Paradoxes arise whenever we try to give classical meaning to every step of the chain. The safe strategy is to give interpretation to the complete experiment and not to its parts. The wave function goes partially through one hole and partially through another. But the particle, meant as the sample of the statistical ensemble, goes always through only one of them. Beyond this safe strategy Quantum Mechanics becomes difficult to understand. But it works!

We decided to publish the paper by J. Lee because it proposes yet another way of thinking about these paradoxes. It is not entirely new: this kind of analysis was always present among those who think about foundations of Quantum Mechanics. Nevertheless, we found it useful to remind our readers that the things are not so simple ... 